Article

\title{
Convex Aggregation Operators and Their Applications to Multi-Hesitant Fuzzy Multi-Criteria Decision-Making
}

\author{
Ye Mei ${ }^{1}$, Juanjuan Peng ${ }^{1}$ and Junjie Yang ${ }^{2, *}$ \\ 1 School of Electrical and Information Engineering, Hubei University of Automotive Technology, \\ Shiyan 442002, China; meiye13635722832@163.com (Y.M.); xiaqing1981@126.com (J.P.) \\ 2 School of Information Engineering, Lingnan Normal University, Zhanjiang 524048, China \\ * Correspondence: yangjunjie1998@lingnan.edu.cn
}

Received: 23 May 2018; Accepted: 25 July 2018; Published: 21 August 2018

\begin{abstract}
Hesitant fuzzy sets (HFSs), which were generalized from fuzzy sets, constrain the membership degree of an element to be a set of possible values between zero and one; furthermore, if two or more decision-makers select the same value, it is only counted once. However, a situation where the evaluation value is repeated several times differs from one where the value appears only once. Multi-hesitant fuzzy sets (MHFSs) can deal effectively with a case where some values are repeated more than once in a MHFS. In this paper, the novel convex combination of multi-hesitant fuzzy numbers (MHFNs) is introduced. Some aggregation operators based on convex operation, such as generalized multi-hesitant fuzzy ordered weighted average (GMHFOWA) operator, generalized multi-hesitant fuzzy hybrid weighted average (GMHFHWA) operator, generalized multi-hesitant fuzzy prioritized weighted average (GMHFPWA) operator and generalized multi-hesitant fuzzy Choquet integral weighted average (GMHFCIWA) operator, are developed and corresponding properties are discussed in detail. Then, based on the proposed aggregation operators, a novel approach for multi-criteria decision-making (MCDM) problem is proposed for ranking alternatives. Finally, an example is provided to verify the developed approach and demonstrate its validity and feasibility and the study is supported by a sensitivity analysis and a comparison analysis.
\end{abstract}

Keywords: multi-criteria decision-making; multi-hesitant fuzzy sets; aggregation operators

\section{Introduction}

Hesitant fuzzy sets (HFSs) and multi-hesitant fuzzy sets (MHFSs), which were originally defined by Torra [1,2], are an extension of Zadeh's fuzzy sets (FSs) [3]. They allow a membership degree to have different possible precise values between zero and one. Recently, HFSs and its extensions has been the subject of a great deal of research and have been widely applied to multi-criteria decision-making (MCDM) problems [4-20]. For example, some works on the aggregation operators of HFSs have been undertaken [9-15] and the correlation coefficient, distance and correlation measures for HFSs were developed [16-20]. For example, Zhang et al. [13] developed some induced generalized hesitant fuzzy operators and applied them to multi-criteria group decision-making (MCGDM) problems. Zhou [14] proposed hesitant fuzzy ordered accurate weighted averaging (HFOAWA) operator and hesitant fuzzy ordered accurate weighted geometric (HFOAWG) operators and applied them to project investment. Zhang [15] defined generalized hesitant fuzzy power average (GHFPA) operator and generalized hesitant fuzzy power geometric (GHFPG) operator and applied them to MCGDM problems. $\mathrm{Yu}$ [16] proposed some aggregation operators based on Einstein operations and applied them to MCDM problems. Wang et al. [21] proposed a wide range of hesitant multiplicative fuzzy power aggregation 
geometric operators on MCGDM problems for hesitant multiplicative information. Torres et al. [22] propose a prioritized aggregation operator to combine a time sequence of hesitant fuzzy information, and applied them to the service selection problem in service-based systems. Qian and Wang [23] generalized HFSs and utilized the aggregation operators to solve MCDM problems. Meng et al. [24] developed some induced generalized hesitant fuzzy Shapley hybrid operators and applied them to MCDM problems. Zhou and $\mathrm{Xu}$ [25] developed an optimal discrete fitting aggregation MCDM method with HFSs. Tan et al. [26] defined some hesitant fuzzy Hamacher aggregation operators and applied them to MCDM problems. Meng and Chen [27] and Liao et al. [28] defined novel correlation coefficients between HFSs and applied them to MCDM problems. Li et al. [29] and Hu et al. [30] defined some new distance and similarity measures of HFSs and applied them to MCDM problems. Furthermore, Zhang and Wei [31] developed the E-VIKOR method to solve MCDM problems with HFSs. Zhang and $\mathrm{Xu}$ [32] proposed the TODIM method, which was based on measured functions with HFSs. Farhadinia $[33,34]$ developed some information measures of HFSs and a novel method of ranking hesitant fuzzy values. Moreover, Peng et al. [35] developed an extension of ELECTRE III method to handle MCDM problems with MHFSs.

However, two main shortcomings of the existing methods of dealing with HFSs have emerged from the research to date. (1) Both distance measures, similarity measures and some comparison methods should satisfy the condition that all hesitant fuzzy numbers (HFN) must be arranged in ascending order and be of equal length. If the two HFNs being compared have different lengths, then the value of the shorter one should be increased until both are equal. However, in such cases, different methods of extension could produce different results. (2) The existing methods do not clarify: how to solve a situation where there is a repeated value in the evaluation of alternatives; and, in particular, whether decision-makers can give more than one value (possible membership degrees of an element) for each criterion or not. At the same time, the situation where the evaluation value is repeated more than once is actually different from that where a value appears only once. For example, decision-makers can determine that the possible degrees of membership by which an alternative is assessed relative to the criterion "excellence" are $0.7,0.8$, and 0.8 , which is expressed in the form of an HFN as $\{0.7,0.8\}$. However, the nature of the evaluation $\{0.7,0.8\}$ substantially differs from that expressed in the form of an MHFS as $\{0.7,0.8,0.8\}$, which can lead to loss of information during the data collection process. Therefore, MHFSs can overcome these shortcomings and deal with the case where some values may be repeated more than once in an HFS. In this paper, the novel MCDM approach is developed based on some convex aggregation operators of multi-hesitant fuzzy sets (MHFNs). Moreover, the proposed approach based on convex operators distinguished from other methods for MCDM method not only because the proposed approach uses MHFSs, but also due to the consideration the inter-dependent phenomena among the criteria, which makes it more consistent with the practical decision-making environment.

The remainder of this paper is organized as follows. In Section 2, the definition, as well as the comparison method, of HFSs and MHFSs is provided. In Section 3, some aggregation operators of multi-hesitant fuzzy numbers (MHFNs) based on convex operation are developed and corresponding properties are discussed. In Section 4, an MCDM method based on convex aggregation operators is proposed. In Section 5, an example to illustrate the practical application of the developed approach is provided as well as sensitivity analysis and comparison analysis. Finally, some conclusions are drawn in Section 6.

\section{Hesitant Fuzzy Sets and Multi-Hesitant Fuzzy Sets}

In this section, the definition of HFSs and MHFSs are reviewed. The comparison method of HFSs, which will be utilized in the latter analysis, are also presented.

Definition 1. Let $X$ be a reference set, and a HFS $E$ on $X$ be in terms of a function which will return a subset of $[0,1]$ in the case of it being applied to $X[1,2]$. 
In order that it would be easily understood, Xia and $\mathrm{Xu}$ [9] expressed the HFS as a mathematical symbol:

$$
E=\left\{\left\langle x, h_{E}(x)\right\rangle \mid x \in X\right\}
$$

where $h_{E}(x)$ is a set of values in [0,1], denoting the possible membership degrees of the element $x \in X$ to the set $E$. E is called HFSs, $h_{E}(x)$ is called a hesitant fuzzy element (HFE) [4], and $H$ is the set of all HFEs. In particular, if $X$ has only one element, $E$ is called a HFN, which can be denoted by $E=\left\{h_{E}(x)\right\}$. The set of all HFNs is represented by HFNS.

Torra [1,2] defined some operations on HFNs, and Xia and Xu [4] defined some new operations on HFNs as well as the score functions.

Definition 2. Let $X$ be a reference set, and MHFSs be defined as $E_{M}$ in terms of a function $H_{E_{M}}$ that returns a multi-subset of $[0,1]$ when applied to $X[1]$.

Based on Definition 1, MHFSs can be expressed by the mathematical equation:

$$
E_{M}=\left\{\left\langle x, H_{E_{M}}(x)\right\rangle \mid x \in X\right\}
$$

Here, $H_{E_{M}}(x)$ is a set of values in $[0,1]$ denoting the possible degrees of membership of the element $x \in X$ to the set $E_{M}$. In any $H_{E_{M}}(x)$, the values can be repeated multiple times. $H_{E_{M}}(x)$ is a multi-hesitant fuzzy element (MHFE), and $H_{E_{M}}$ is the set of all MHFEs. It is noteworthy that, if $X$ contains only a single element, $E_{M}$ is called a MHFN, briefly denoted by $E_{M}=\left\{H_{E_{M}}(x)\right\}$. The set of all MHFNs is represented by MHFNS. Any HFS is a special case of an MHFS.

Moreover, the operations of HFNs between two HFNs $H_{A}$ and $H_{B}$ on $X$ was defined as below [1]:

(1) $\lambda H_{A}=\left\{1-\left(1-\gamma_{H_{A}}\right)^{\lambda}\right\}(\lambda>0)$

(2) $H_{A}^{\lambda}=\left\{\left(\gamma_{H_{A}}\right)^{\lambda}\right\}(\lambda>0)$;

(3) $H_{A} \oplus H_{B}=\left\{\gamma_{H_{A}}+\gamma_{H_{B}}-\gamma_{H_{A}} \cdot \gamma_{H_{B}}\right\}$;

(4) $H_{A} \otimes H_{B}=\left\{\gamma_{H_{A}} \cdot \gamma_{H_{B}}\right\}$.

Apparently, the operations on HFNs presented in Definition 2 also can be suitable for MHFNs.

The ranking of two HFNs can be obtained by combining the score function and the accuracy function $[9,36]$.

Definition 3. Let $H_{A}$ and $H_{B}$ be two HFNs on $X$, and then the novel ranking method for MHFNs can be defined as follows [36]:

(1) if $s\left(H_{A}\right)<s\left(H_{B}\right)$, then $H_{A} \prec H_{B}$;

(2) if $s\left(H_{A}\right)=s\left(H_{B}\right)$, then:

- if $f\left(H_{A}\right)=f\left(H_{B}\right)$, then $H_{A} \sim H_{B}$;

- if $f\left(H_{A}\right)<f\left(H_{B}\right)$, then $H_{A} \succ H_{B}$;

- if $f\left(H_{A}\right)>f\left(H_{B}\right)$, then $H_{A} \prec H_{B}$.

where $s\left(H_{i}\right)=\frac{1}{l_{H_{i}}} \sum_{\gamma_{i} \in H_{i}} \gamma_{i}$ and $f\left(H_{i}\right)=\frac{1}{l_{H_{i}}-1} \sum_{\gamma_{i} \in H_{i}}\left(s\left(H_{i}\right)-\gamma_{i}\right)^{2}(i=A, B)$ represents the score function and accuracy function of $H_{i}$ respectively [4,31], and $l_{H_{i}}$ is the number of elements in $H_{i}$. Please note that " $\prec$ " means "inferior to". The score function is similar to the mean value; the greater the value of the mean, the larger the hesitant degree. The accuracy function is similar to the sample variance in statistics and can reflect the fluctuation of evaluation values of HFNs; the greater the amplitude of fluctuation, the larger the hesitant degree. 
Example 1. Let $h_{1}=\{0.2,0.5\}$ and $h_{2}=\{0.3,0.4\}$ be two HFNs. According to Definition 3 , we have $s\left(h_{1}\right)=s\left(h_{2}\right)=0.35$ and $f\left(h_{1}\right)=0.045>f\left(h_{2}\right)=0.005$. Apparently, $H_{1} \prec H_{2}$ can be obtained, which is consistent with our intuitive.

\section{The Convex Combination Operation and Some Aggregation Operators of MHFNs}

In this section, the convex combination operation with MHFNs is developed, and corresponding properties and aggregation operators are presented.

Definition 4. Let $\mathrm{H}_{1}$ and $\mathrm{H}_{2}$ be two MHFNs. A convex combination of $H_{1}$ and $\mathrm{H}_{2}$ is defined as

$$
C^{2}\left(w_{1}, \mathrm{H}_{1}, w_{2}, H_{2}\right)=w_{1} \otimes H_{1} \oplus w_{2} \otimes H_{2}=\left\{\left(w_{1} \gamma_{1}^{\lambda}+w_{2} \gamma_{2}^{\lambda}\right)^{1 / \lambda} \mid \gamma_{1} \in H_{1}, \gamma_{2} \in H_{2}\right\}, \lambda>0
$$

where $w_{1} \geq 0, w_{2} \geq 0$ and $w_{1}+w_{2}=1$.

Proposition 1. Let $H_{1}$ and $H_{2}$ be two MHFNs. For $0 \leq w \leq 1$, the convex combination $C^{2}\left(w, \mathrm{H}_{1}, 1-w, H_{2}\right)$ of $H_{1}$ and $H_{2}$ is also a MHFNs.

Proof. Based on Definition 4, we just need to prove that $0<\left(w \gamma_{1}^{\lambda}+(1-w) \gamma_{2}^{\lambda}\right)^{1 / \lambda} \leq 1$. It is obvious that $\left(w \gamma_{1}^{\lambda}+(1-w) \gamma_{2}^{\lambda}\right)^{1 / \lambda}>0$. Assume $\gamma_{1}^{\prime} \in H_{1}$ and $\gamma_{2}^{\prime} \in H_{2}$ do exist, so to make $\left(w \gamma_{1}^{\prime \lambda}+(1-w) \gamma_{2}^{\prime \lambda}\right)^{1 / \lambda} \geq 1$ i.e., $w \gamma_{1}^{\prime \lambda}+(1-w) \gamma_{2}^{\prime \lambda} \geq 1$. If $\gamma_{1}^{\prime}>\gamma_{2}^{\prime}$, then $w \gamma_{1}^{\prime \lambda}+(1-w) \gamma_{2}^{\prime \lambda} \geq 1$ i.e., $w>\frac{1-\gamma_{2}^{\prime \lambda}}{\gamma_{1}^{\prime \lambda}-\gamma_{2}^{\prime \lambda}}>1$ which obviously contradicts $0 \leq w \leq 1$; if $\gamma_{1}^{\prime}=\gamma_{2}^{\prime}$, then $w \gamma_{1}^{\prime \lambda}+(1-w) \gamma_{1}^{\prime \lambda}=\gamma_{1}^{\prime \lambda}>1$ which contradicts $0<\gamma_{1}^{\prime \lambda} \leq 1$; if $\gamma_{1}^{\prime} \leq \gamma_{2}^{\prime}$, then $w \gamma_{1}^{\prime \lambda}+(1-w) \gamma_{2}^{\prime \lambda} \geq 1$ i.e., $w<\frac{1-\gamma_{2}^{\prime \lambda}}{\gamma_{1}^{\prime \lambda}-\gamma_{2}^{\prime \prime}}<0$ which obviously contradicts $0 \leq w \leq 1$. Therefore, the hypothesis is not supported. For any $\gamma_{1} \in H_{1}$ and $\gamma_{2} \in H_{2}$, we have $\left(w \gamma_{1}^{\lambda}+(1-w) \gamma_{2}^{\lambda}\right)^{1 / \lambda} \leq 1$. Thus, the convex combination $C^{2}\left(w, \mathrm{H}_{1}, 1-w, H_{2}\right)$ of $H_{1}$ and $H_{2}$ is also a MHFNs.

Definition 5. Let $H_{i}(i=1,2, \ldots, n)$ be a collection of MHFNs. Then the generalized multi-hesitant fuzzy ordered weighted average (GMHFOWA) operator of dimension $n$ is a mapping GMHFOWA : MHFN ${ }^{n} \rightarrow$ MHFN that has an associated weight vector $w=\left(w_{1}, w_{2}, \ldots, w_{n}\right)$ with $w_{i} \geq 0$ $(i=1,2, \ldots, n)$ and $\sum_{i=1}^{n} w_{i}=1$, and

$$
\begin{aligned}
& \operatorname{GMHFOWA}\left(H_{1}, H_{2}, \ldots, H_{n}\right) \\
& =C^{n}\left(w_{k}, H_{\sigma(k)}, k=1,2, \ldots, n\right)=w_{1} \otimes H_{\sigma(1)} \oplus\left(1-w_{1}\right) \otimes C^{n-1}\left\{w_{i} / \sum_{k=2}^{n} w_{k}, H_{\sigma(i)}, i=2,3, \ldots, n\right\} .
\end{aligned}
$$
$H_{\sigma(n)}$.

Here $(\sigma(1), \sigma(2), \ldots, \sigma(n))$ is a permutation of $(1,2, \ldots, n)$, and such that $H_{\sigma(1)} \leq H_{\sigma(2)} \leq \ldots \leq$

Theorem 1. Let $H_{i}(i=1,2, \ldots, n)$ be a collection of MHFNs. Then their aggregated value by using the GMHFOWA operator is also a MHFN, and

$$
\operatorname{GMHFOWA}\left(H_{1}, H_{2}, \ldots, H_{n}\right)=\left\{\left(w_{1} \gamma_{\sigma(1)}^{\lambda}+w_{2} \gamma_{\sigma(2)}^{\lambda}+\ldots+w_{n} \gamma_{\sigma(n)}^{\lambda}\right)^{1 / \lambda} \mid \gamma_{\sigma(i)} \in H_{\sigma(i)}, i=1,2, \ldots, n\right\}
$$

Here $(\sigma(1), \sigma(2), \ldots, \sigma(n))$ is a permutation of $(1,2, \ldots, n)$, and such that $H_{\sigma(1)} \leq H_{\sigma(2)} \leq \ldots \leq$ $H_{\sigma(n)}$. 
Example 2. Let $H_{1}=\{0.2,0.2,0.3\}, H_{2}=\{0.1,0.2\}$ and $H_{3}=\{0.4\}$ be three MHFNs, $w=(0.3,0.4,0.3)$ be the weight vector of them, and $\lambda=1$. Based on Definition 5 and Theorem 1, if the associated vector is $w=(0.3,0.4,0.3)$, then $H_{3}>H_{1}>H_{2}, H_{\sigma(1)}=H_{2}, H_{\sigma(2)}=H_{1}$ and $H_{\sigma(3)}=H_{3}$ can be obtained. So

$$
\begin{aligned}
& \operatorname{GMHFOWA}\left(H_{1}, H_{2}, H_{3}\right) \\
& =\left\{w_{1} \gamma_{2}+w_{2} \gamma_{1}+w_{3} \gamma_{3} \mid \gamma_{1} \in H_{1}, \gamma_{2} \in H_{2}, \gamma_{3} \in H_{3}\right\}=\{0.23,0.23,0.27,0.26,0.26,0.30\}
\end{aligned}
$$

It can be easily proved that the GMHFOWA operator is monotonicity, commutativity and bounded, which are presented in the following.

Proposition 2. Let $H_{i}(i=1,2, \ldots, n)$ be a collection of MHFNs, the following prosperities can be true.

(1) (Monotonicity) Let $H_{i}^{\prime}(i=1,2, \ldots, n)$ be a collection of MHFNs. If for all $i, H_{i} \leq H_{i}^{\prime}$, then

$$
\operatorname{GMHFOWA}\left(H_{1}, H_{2}, \ldots, H_{n}\right) \leq \operatorname{GMHFOWA}\left(H_{1}^{\prime}, H_{2}^{\prime}, \ldots, H_{n}^{\prime}\right)
$$

(2) (Commutativity) If $H_{1}^{*}, \ldots, H_{n}^{*}$ is a permutation of $H_{1}, \ldots, H_{n}$, then

$$
\operatorname{GMHFOWA}\left(H_{1}^{*}, H_{2}^{*}, \ldots, H_{n}^{*}\right)=\operatorname{GMHFOWA}\left(H_{1}, H_{2}, \ldots, H_{n}\right)
$$

(3) (Boundedness) If $H^{-}=\left\{\gamma_{1}^{-}, \gamma_{2}^{-}, \ldots, \gamma_{n}^{-}\right\}$and $H^{+}=\left\{\gamma_{1}^{+}, \gamma_{2}^{+}, \ldots, \gamma_{n}^{+}\right\}$, where $\gamma_{i}^{-}=\min _{\gamma_{i} \in H_{i}} \gamma_{i}$ and $\gamma_{i}^{+}=\max _{\gamma_{i} \in H_{i}} \gamma_{i}$, then

$$
H^{-} \leq \operatorname{GMHFOWA}\left(H_{1}, H_{2}, \ldots, H_{n}\right) \leq H^{+}
$$

Definition 6. Let $H_{i}(i=1,2, \ldots, n)$ be a collection of MHFNs. Then the generalized multi-hesitant fuzzy hybrid weighted average (GMHFHWA) operator of dimension $n$ is a mapping GMHFHWA : MHFN ${ }^{n} \rightarrow$ MHFN that have the weighting vector $w=\left(w_{1}, w_{2}, \ldots, w_{n}\right)$ of $H_{i}(i=1,2, \ldots, n)$ with $w_{i} \geq 0(i=1,2, \ldots, n)$ and $\sum_{i=1}^{n} w_{i}=1$, the aggregation-associated vector is $\omega=\left(\omega_{1}, \omega_{2}, \ldots, \omega_{n}\right)$ with $\omega_{i} \geq 0(i=1,2, \ldots, n)$ and $\sum_{i=1}^{n} \omega_{i}=1$, and

$$
\begin{aligned}
& \operatorname{GMHFHWA}\left(H_{1}, H_{2}, \ldots, H_{n}\right) \\
& =C^{n}\left(\omega_{k}, \dot{H}_{\sigma(k)}, k=1,2, \ldots, n\right)=\omega_{1} \otimes \dot{H}_{\sigma(1)} \oplus\left(1-\omega_{1}\right) \otimes C^{n-1}\left\{\omega_{i} / \sum_{k=2}^{n} \omega_{k}, \dot{H}_{\sigma(i)}, i=2,3, \ldots, n\right\} .
\end{aligned}
$$

Here $(\sigma(1), \sigma(2), \ldots, \sigma(n))$ is a permutation of $(1,2, \ldots, n)$, and $\dot{H}_{\sigma(i)}=n w_{i} H_{i}(i=1,2, \ldots, n)$ is the $i$-th largest of the weighted multi-hesitant fuzzy values. $n$ is the balancing coefficient which plays a role of balance. If $\omega=(1 / n, 1 / n, \ldots, 1 / n)$, then the GMHFHWA operator is reduced to the GMHFOWA operator. If $w=(1 / n, 1 / n, \ldots, 1 / n)$, then the GMHFHWA operator is reduced to the GMHFWA operator.

Theorem 2. Let $H_{i}(i=1,2, \ldots, n)$ be a collection of MHFNs. Then their aggregated value by using GMHFHWA operator is also a MHFN, and

$$
\operatorname{GMHFHWA}\left(H_{1}, H_{2}, \ldots, H_{n}\right)=\left\{\left(\omega_{1} \dot{\gamma}_{\sigma(1)}^{\lambda}+\omega_{2} \dot{\gamma}_{\sigma(2)}^{\lambda}+\ldots+\omega_{n} \dot{\gamma}_{\sigma(n)}^{\lambda}\right)^{1 / \lambda} \mid \dot{\gamma}_{\sigma(i)} \in \dot{H}_{\sigma(i)}, i=1,2, \ldots, n\right\}
$$

Here $(\sigma(1), \sigma(2), \ldots, \sigma(n))$ is a permutation of $(1,2, \ldots, n)$, and $\dot{H}_{\sigma(i)}=n w_{i} H_{i}(i=1,2, \ldots, n)$ is the $i$-th largest of the weighted multi-hesitant fuzzy values. $n$ is the balancing coefficient which plays a role of balance. 
Example 3. Let $H_{1}=\{0.2,0.2\}, H_{2}=\{0.1,0.3\}$ and $H_{3}=\{0.4\}$ be three MHFNs. The weight vector is $w=(0.3,0.4,0.3)$ and aggregation-associated vector is also $\omega=(0.2,0.4,0.4)$, and $\lambda=1$, then

$\dot{H}_{1}=3 \times 0.3 \cdot H_{1}=\{0.1819,0.1819\} ; \dot{H}_{2}=3 \times 0.4 \cdot H_{2}=\{0.0126,0.0419\} ; \dot{H}_{3}=3 \times 0.3 \cdot H_{3}=\{0.3686\}$.

Obviously, $s\left(\dot{H}_{3}\right)>s\left(\dot{H}_{1}\right)>s\left(\dot{H}_{2}\right)$. By using Theorem 3, we have

$\operatorname{GMHFWA}\left(H_{1}, H_{2}, H_{3}\right)=\left\{\left(\omega_{1} \dot{\gamma}_{\sigma(1)}+\omega_{2} \dot{\gamma}_{\sigma(2)}+\ldots+\omega_{n} \dot{\gamma}_{\sigma(n)}\right) \mid \dot{\gamma}_{\sigma(i)} \in \dot{H}_{\sigma(i)}, i=1,2,3\right\}$

$=\{0.1512,0.1632,0.1512,0.1632\}$.

Proposition 3. Let $H_{i}(i=1,2, \ldots, n)$ be a collection of MHFNs, the following prosperities can be true.

(1) (Monotonicity) Let $H_{i}^{\prime}(i=1,2, \ldots, n)$ be a collection of MHFNs. If for all $i, H_{i} \leq H_{i}^{\prime}$, then

$$
\operatorname{GMHFHWA}\left(H_{1}, H_{2}, \ldots, H_{n}\right) \leq \operatorname{GMHFHWA}\left(H_{1}^{\prime}, H_{2}^{\prime}, \ldots, H_{n}^{\prime}\right)
$$

(2) (Commutativity) If $H_{1}^{*}, \ldots, H_{n}^{*}$ is a permutation of $H_{1}, \ldots, H_{n}$, then

$$
\operatorname{GMHFHWA}\left(H_{1}^{*}, H_{2}^{*}, \ldots, H_{n}^{*}\right)=\operatorname{GMHFHWA}\left(H_{1}, H_{2}, \ldots, H_{n}\right)
$$

(3) (Boundedness) If $H^{-}=\left\{\gamma_{1}^{-}, \gamma_{2}^{-}, \ldots, \gamma_{n}^{-}\right\}$and $H^{+}=\left\{\gamma_{1}^{+}, \gamma_{2}^{+}, \ldots, \gamma_{n}^{+}\right\}$, where $\gamma_{i}^{-}=\min _{\gamma_{i} \in H_{i}} \gamma_{i}$ and $\gamma_{i}^{+}=\max _{\gamma_{i} \in H_{i}} \gamma_{i}$, then

$$
H^{-} \leq \operatorname{GMHFHWA}\left(H_{1}, H_{2}, \ldots, H_{n}\right) \leq H^{+}
$$

Based on the prioritization between the criteria discussed in [37], the prioritized aggregation operator can be obtained.

Definition 7. Let $H_{i}(i=1,2, \ldots, n)$ be a collection of MHFNs. Then the generalized multi-hesitant fuzzy prioritized weighted average (GMHFPWA) operator of dimension $n$ is a mapping GMHFPWA : MHFN ${ }^{n} \rightarrow$ MHFN, and

$$
\begin{aligned}
& \operatorname{GMHFPWA}\left(H_{1}, H_{2}, \ldots, H_{n}\right) \\
& =C^{n}\left(\frac{T_{k}}{\sum_{j=1}^{n} T_{j}}, H_{k}, k=1,2, \ldots, n\right) \\
& =\frac{T_{1}}{\sum_{j=1}^{n} T_{j}} \otimes H_{1} \oplus\left(1-\frac{T_{1}}{\sum_{j=1}^{n} T_{j}}\right) \otimes C^{n-1}\left\{\frac{T_{i}}{\sum_{j=1}^{n} T_{j}} / \sum_{k=2}^{n} \frac{T_{k}}{\sum_{j=1}^{n} T_{j}}, H_{i}, i=2,3, \ldots, n\right\} .
\end{aligned}
$$

Here $T_{j}=\prod_{k=1}^{j-1} s\left(H_{k}\right)(j=2, \ldots, n), T_{1}=1$ and $s\left(H_{k}\right)$ is the score values of $H_{k}(k=1,2, \ldots, n)$.

Theorem 3. Let $H_{i}(i=1,2, \ldots, n)$ be a collection of MHFNs. Then their aggregated value by using the GMHFPWA operator is also a MHFN, and

$$
\operatorname{GMHFPWA}\left(H_{1}, H_{2}, \ldots, H_{n}\right)=\left\{\left(\frac{T_{1}}{\sum_{j=1}^{n} T_{j}} \gamma_{1}^{\lambda}+\frac{T_{2}}{\sum_{j=1}^{n} T_{j}} \gamma_{2}^{\lambda}+\ldots+\frac{T_{n}}{\sum_{j=1}^{n} T_{j}} \gamma_{n}^{\lambda}\right)^{1 / \lambda} \mid \gamma_{i} \in H_{i}, i=1,2, \ldots, n\right\}
$$

Here $T_{j}=\prod_{k=1}^{j-1} s\left(H_{k}\right)(j=2, \ldots, n), T_{1}=1$ and $s\left(H_{k}\right)$ is the score values of $H_{k}(k=1,2, \ldots, n)$.

Example 4. Let $H_{1}=\{0.5,0.5,0.7\}, H_{2}=\{0.4,0.5\}, H_{3}=\{0.8,0.9\}$ and $H_{4}=\{0.3,0.4,0.5\}$ be four MHFNs and $\lambda=1$. Based on Definition 3, then $s\left(H_{1}\right)=0.567, s\left(H_{2}\right)=0.45$ and $s\left(H_{3}\right)=0.85$ can be 
obtained. If the prioritization during four MHFNs is $H_{1} \succ H_{2} \succ H_{3} \succ H_{4}$, then according to Definition 7 and Theorem 3,

$$
T_{41}=1, T_{2}=s\left(H_{1}\right)=0.567, T_{3}=s\left(H_{1}\right) \times s\left(H_{2}\right)=0.2552, T_{4}=0.2169, \sum_{j=1}^{4} T_{j}=2.0391 .
$$

So

GMHFPWA $\left(H_{1}, H_{2}, H_{3}, H_{4}\right)=\left\{\left(\frac{1}{2.0391} \gamma_{1}+\frac{0.567}{2.0391} \gamma_{2}+\frac{0.2552}{2.0391} \gamma_{3}+\frac{0.2169}{2.0391} \gamma_{4}\right) \mid \gamma_{i} \in H_{i}, i=1,2,3,4\right\}$

$=\{0.4885,0.4991,0.5097,0.5010,0.5116,0.5223,0.5163,0.5269,0.5375,0.5288,0.5394,0.5501,0.4885,0.4991$, $0.5097,0.5010,0.5116,0.5223,0.5163,0.5269,0.5375,0.5288,0.5394,0.5501,0.5865,0.5972,0.6078,0.5991$, $0.6097,0.6203,0.6144,0.6250,0.6356,0.6269,0.6375,0.6481\}$.

Similarly, it can be easily proved that the GMHFPWA operator is monotonicity, commutativity and bounded, which are presented in the following.

Proposition 4. Let $H_{i}(i=1,2, \ldots, n)$ be a collection of MHFNs, then the following properties can be true.

(1) (Monotonicity) Let $H_{i}^{\prime}(i=1,2, \ldots, n)$ be a collection of MHFNs. If for all $i, H_{i} \leq H_{i}^{\prime}$, then

$$
\operatorname{GMHFPWA}\left(H_{1}, H_{2}, \ldots, H_{n}\right) \leq \operatorname{GMHFPWA}\left(H_{1}^{\prime}, H_{2}^{\prime}, \ldots, H_{n}^{\prime}\right)
$$

(2) (Commutativity) If $H_{1}^{*}, \ldots, H_{n}^{*}$ is a permutation of $H_{1}, \ldots, H_{n}$, then

$$
\operatorname{GMHFPWA}\left(H_{1}^{*}, H_{2}^{*}, \ldots, H_{n}^{*}\right)=\operatorname{GMHFPWA}\left(H_{1}, H_{2}, \ldots, H_{n}\right)
$$

(3) (Boundedness) If $H^{-}=\left\{\gamma_{1}^{-}, \gamma_{2}^{-}, \ldots, \gamma_{n}^{-}\right\}$and $H^{+}=\left\{\gamma_{1}^{+}, \gamma_{2}^{+}, \ldots, \gamma_{n}^{+}\right\}$, where $\gamma_{i}^{-}=\min _{\gamma_{i} \in H_{i}} \gamma_{i}$ and $\gamma_{i}^{+}=\max _{\gamma_{i} \in H_{i}} \gamma_{i}$, then

$$
H^{-} \leq \operatorname{GMHFPWA}\left(H_{1}, H_{2}, \ldots, H_{n}\right) \leq H^{+}
$$

According to the fuzzy measure (more details can be founded in [38]), the Choquet integral aggregation operator can be obtained.

Definition 8. Let $H_{i}(i=1,2, \ldots, n)$ be a collection of MHFNs. Then the generalized multi-hesitant fuzzy Choquet integral weighted average (GMHFCIWA) operator of dimension $n$ is a mapping GMHFCIWA : MHFN ${ }^{n} \rightarrow$ MHFN, and

$$
\begin{aligned}
& \operatorname{GMHFCIWA}\left(H_{1}, H_{2}, \ldots, H_{n}\right) \\
& =C^{n}\left(\left(\mu\left(A_{\sigma(k)}\right)-\mu\left(A_{\sigma(k+1)}\right)\right), H_{\sigma(k)}, k=1,2, \ldots, n\right) \\
& =\left(\mu\left(A_{\sigma(1)}\right)-\mu\left(A_{\sigma(2)}\right)\right) \otimes H_{\sigma(1)} \oplus\left(1-\left(\mu\left(A_{\sigma(1)}\right)-\mu\left(A_{\sigma(2)}\right)\right)\right) \\
& \otimes C^{n-1}\left\{\left(\left(\mu\left(A_{\sigma(i)}\right)-\mu\left(A_{\sigma(i+1)}\right)\right) / \sum_{k=2}^{n}\left(\mu\left(A_{\sigma(k)}\right)-\mu\left(A_{\sigma(k+1)}\right)\right), H_{\sigma(i)}, i=2,3, \ldots, n\right)\right\} .
\end{aligned}
$$

Here $\mu$ is a fuzzy measure on $X,(\sigma(1), \sigma(2), \ldots, \sigma(n))$ is a permutation of $(1,2, \ldots, n)$, and such that $H_{\sigma(1)} \leq H_{\sigma(2)} \leq \ldots \leq H_{\sigma(n)} . A_{\sigma(i)}=\left\{x_{\sigma(k)} \mid k \geq i\right\}$, and $x_{\sigma(i)}$ is the criterion corresponding to $H_{\sigma(i)}$.

Theorem 4. Let $H_{i}(i=1,2, \ldots, n)$ be a collection of MHFNs, then their aggregated value by using the GMHFCIWA operator is also a MHFN, and

$$
\begin{aligned}
& \operatorname{GMHFCIWA}\left(H_{1}, H_{2}, \ldots, H_{n}\right) \\
& =\left\{\left(\left(\mu\left(A_{\sigma(1)}\right)-\mu\left(A_{\sigma(2)}\right)\right) \gamma_{\sigma(1)}^{\lambda}+\left(\mu\left(A_{\sigma(2)}\right)-\mu\left(A_{\sigma(3)}\right)\right) \gamma_{\sigma(2)}^{\lambda}+\ldots+\left(\mu\left(A_{\sigma(n)}\right)-\mu\left(A_{\sigma(n+1)}\right)\right) \gamma_{\sigma(n)}^{\lambda}\right)^{1 / \lambda}\right. \\
& \left.\mid \gamma_{\sigma(i)} \in H_{\sigma(i)}, i=1,2, \ldots, n\right\} .
\end{aligned}
$$


Here $(\sigma(1), \sigma(2), \ldots, \sigma(n))$ is a permutation of $(1,2, \ldots, n)$, and such that $H_{\sigma(1)} \leq H_{\sigma(2)} \leq \ldots \leq$ $H_{\sigma(n)} \cdot A_{\sigma(i)}=\left\{x_{\sigma(k)} \mid k \geq i\right\}$, and $x_{\sigma(i)}$ is the criterion corresponding to $H_{\sigma(i)}$.

Example 5. Suppose a Venture Capital Company is going to evaluate the existed investment projects from the financial perspective. Three criteria could be considered: $c_{1}$ : operating capacity; $c_{2}$ : solvency; $c_{3}$ : profitability. Suppose three criteria are inter-dependent. The decision-makers could give the evaluation values in form of MHFNs and denoted as follows: $H_{1}=\{0.1,0.1\}, H_{2}=\{0.2,0.4\}$ and $H_{3}=\{0.5\}$.

Suppose that $\mu\left(c_{1}\right)=0.30, \mu\left(c_{2}\right)=0.30, \mu\left(c_{3}\right)=0.20, \mu\left(c_{1}, c_{2}\right)=0.90, \mu\left(c_{1}, c_{3}\right)=0.80$, $\mu\left(c_{2}, c_{3}\right)=0.60, \mu\left(c_{1}, c_{2}, c_{3}\right)=1$, then the following results can be obtained.

$$
\begin{aligned}
w_{\sigma(1)} & =\mu\left(A_{\sigma(1)}\right)-\mu\left(A_{\sigma(2)}\right) \\
& =\mu\left(c_{\sigma(1)}, c_{\sigma(2)}, c_{\sigma(3)}\right)-\mu\left(c_{\sigma(2)}, c_{\sigma(3)}\right)=\mu\left(c_{1}, c_{2}, c_{3}\right)-\mu\left(c_{1}, c_{3}\right)=1-0.80=0.20 ; \\
w_{\sigma(2)}= & 0.60 ; w_{\sigma(3)}=0.20 .
\end{aligned}
$$

Then $H_{\sigma(1)}=H_{2}, H_{\sigma(2)}=H_{1}, H_{\sigma(3)}=H_{3}$.

Thus, the overall evaluation value can be calculated.

$$
\begin{aligned}
& \operatorname{GMHFCIWA}\left(H_{1}, H_{2}, H_{3}\right) \\
& =C^{4}\left(\mu\left(A_{\sigma(k)}\right)-\mu\left(A_{\sigma(k+1)}\right), H_{\sigma(k)}, k=1,2,3\right) \\
& =\left\{w_{\sigma(1)} \gamma_{\sigma(1)}+w_{\sigma(2)} \gamma_{\sigma(2)}+w_{\sigma(3)} \gamma_{\sigma(3)} \mid \gamma_{\sigma(i)} \in H_{\sigma(i)}, i=1,2,3\right\} \\
& =\{0.20,0.20,0.24,0.24\} .
\end{aligned}
$$

Similarly, the GMHFCIWA operator is monotonicity, commutativity and bounded, which are presented in the following.

Proposition 5. Let $H_{i}(i=1,2, \ldots, n)$ be a collection of MHFNs, and $\mu$ be the fuzzy measure on $X$, then the following properties can be true.

(1) (Monotonicity) Let $H_{i}^{\prime}(i=1,2, \ldots, n)$ be a collection of MHFNs. If for all $i, H_{i} \leq H_{i}^{\prime}$, then

$$
\operatorname{GMHFCIWA} A_{\mu}\left(H_{1}, H_{2}, \ldots, H_{n}\right) \leq \operatorname{GMHFCIWA} A_{\mu}\left(H_{1}^{\prime}, H_{2}^{\prime}, \ldots, H_{n}^{\prime}\right)
$$

(2) (Commutativity) If $H_{1}^{*}, \ldots, H_{n}^{*}$ is a permutation of $H_{1}, \ldots, H_{n}$, then

$$
\operatorname{GMHFCIWA} A_{\mu}\left(H_{1}^{*}, H_{2}^{*}, \ldots, H_{n}^{*}\right)=\operatorname{GMHFCIWA} A_{\mu}\left(H_{1}, H_{2}, \ldots, H_{n}\right)
$$

(3) (Boundedness) If $H^{-}=\left\{\gamma_{1}^{-}, \gamma_{2}^{-}, \ldots, \gamma_{n}^{-}\right\}$and $H^{+}=\left\{\gamma_{1}^{+}, \gamma_{2}^{+}, \ldots, \gamma_{n}^{+}\right\}$, where $\gamma_{i}^{-}=\min _{\gamma_{i} \in H_{i}} \gamma_{i}$ and $\gamma_{i}^{+}=\max _{\gamma_{i} \in H_{i}} \gamma_{i}$, then

$$
H^{-} \leq \operatorname{GMHFCIWA} A_{\mu}\left(H_{1}, H_{2}, \ldots, H_{n}\right) \leq H^{+}
$$

\section{The MCDM Method Based on Aggregation Operators with MHFNs}

The MCDM ranking/selection problems with multi-hesitant fuzzy information consists of a group of alternatives, denoted by $A=\left\{a_{1}, a_{2}, \ldots, a_{n}\right\}$. The alternatives are evaluated based on the criteria denoted by $C=\left\{c_{1}, c_{2}, \ldots, c_{m}\right\} . a_{i j}$ is the value of the alternative $a_{i}$ for the criterion $c_{j}$, and $a_{i j}=\left\{\gamma_{i j}^{k}, k=1,2, \ldots, l\left(a_{i j}\right)\right\}(i=1, \ldots, n ; j=1, \ldots, m)$ are in the form of MHFNs, which are given by several decision-makers. Furthermore, $l\left(a_{i j}\right)$ represents the number of elements in $a_{i j}$ and the corresponding weight vector $w=\left(w_{1}, w_{2}, \ldots, w_{m}\right)$. This method is suitable if the number of decision-makers is small. A situation could arise where decision-makers evaluate these alternatives 
based on the given criteria, and one decision-maker could give several evaluation values. In particular, in the case where two or more decision-makers give the same value, it is counted repeatedly. $a_{i j}$ is the set of evaluation values for all decision-makers.

The approach is an integration of MHFNs and aggregation operators to solve MCDM problems mentioned above. It is noted that different operators have different characteristic. The decision-makers can choose different operators according to their preference. The GMHFOWA operator mainly weights the ordered positions of the multi-hesitant fuzzy values instead of weighting the multi-hesitant fuzzy values themselves. The GMHFHWA operator reflects the importance degrees of both multi-hesitant fuzzy values and their ordered positions. Furthermore, most MCDM methods are under the assumption that the criteria are at the same priority level, and the prominent of characteristic of the GMHFPWA is that it considers prioritization among the criteria. The GMHFCIWA operator can better reflect the correlations among the elements to handle MCDM problems where the criteria are inter-dependent or interactive. Therefore, four aggregation operators can be used to deal with different relationships among the aggregated arguments, could handle MCDM problems in a flexible and objective manner under multi-hesitant fuzzy environment, and can provide more choices for decision-makers.

The procedure of this approach is shown as follows.

Step 1. Normalize the decision matrix.

For MCDM problems, the most common criteria are of maximizing and minimizing types. To unify all criteria, it is necessary to normalize the evaluation values. (Note: if all the criteria are of the maximizing type and have the same measurement unit, then there is no need to normalize them). Suppose that the matrix $R=\left(a_{i j}\right)_{n \times m^{\prime}}$ where $a_{i j}=\left\{\gamma_{i j}^{1}, \gamma_{i j}^{2}, \ldots, \gamma_{i j}^{k}\right\}$ $\left(i=1,2, \ldots, n ; j=1,2, \ldots, m ; k=1,2, \ldots, l\left(a_{i j}\right)\right)$, are MHFNs, is normalized into the corresponding matrix $\widetilde{R}=\left(\widetilde{a}_{i j}\right)_{n \times m}$. Where $\widetilde{a}_{i j}=\left\{\widetilde{\gamma}_{i j}^{1}, \widetilde{\gamma}_{i j}^{2}, \ldots, \widetilde{\gamma}_{i j}^{k}\right\}\left(i=1,2, \ldots, n ; j=1,2, \ldots, m ; k=1,2, \ldots, l\left(a_{i j}\right)\right)$. $l\left(a_{i j}\right)$ is the number of the elements of $a_{i j}$.

For the maximizing criteria, the normalization formula is

$$
\widetilde{\gamma}_{i j}^{k}=\gamma_{i j}^{k}, k=1,2, \ldots, l\left(a_{i j}\right)
$$

for the minimizing criteria,

$$
\widetilde{\gamma}_{i j}^{k}=1-\gamma_{i j}^{k}, k=1,2, \ldots, l\left(a_{i j}\right)
$$

Seemingly, the normalization values $\widetilde{a}_{i j}=\left\{\widetilde{\gamma}_{i j^{\prime}}^{1} \widetilde{\gamma}_{i j^{\prime}}^{2}, \ldots, \widetilde{\gamma}_{i j}^{k}\right\}\{i=1,2, \ldots, n ; j=1,2, \ldots, m\}$ are also MHFNs.

Step 2. Aggregate the MHFNs of each decision-maker.

Utilize the GMHFOWA, GMHFHWA, GMHPWA or GMHCIWA operator to aggregate the MHFNs of each decision-maker, and the individual aggregated value $y_{i}$ of the alternative $a_{i}$ $(i=1,2, \ldots, n)$ can be obtained.

Step 3. Calculate the score function value $s\left(y_{i}\right)$ and the accuracy function value $a\left(y_{i}\right)$ of $y_{i}$ $(i=1,2, \ldots, m)$ using Definition 3.

Step 4. Rank the alternatives.

\section{An Illustrative Example}

In this section, an example is adapted from Schmeidler [39] for further illustration of the feasibility of the proposed approach.

There is an investment company, which wants to invest in a project. There are five possible alternatives in which to invest: $a_{1}$ is a car company; $a_{2}$ is a food company; $a_{3}$ is a computer company; $a_{4}$ is an arms company; and $a_{5}$ is a TV company. The investment company must make a decision according to the following four criteria: $c_{1}$ is the environment impact; $c_{2}$ is the risk; $c_{3}$ are the growth prospects; and $c_{4}$ is the social-political impact. The environmental impact refers to the impact on the company's 
environment and the processes used in making the product, such as the management methods and work environment. The risk involves more than one risk factor, including product risk and development environment risk. The growth prospects include increased profitability and returns. The social-political impact refers to the government's and local residents' support for company. The four criteria are correlated with each other in the assessment process. The five possible alternatives $a_{i}(i=1,2, \ldots, 5)$ are to be evaluated using the multi-hesitant fuzzy information of two decision-makers as presented in Table 1 . The evaluation values $a_{i j}(i=1,2,3,4,5 ; j=1,2,3,4)$ should be in the form of MHFNs which are provided by two decision-makers based on their knowledge and experience. In the case where decision-makers give the same value, then it is counted repeatedly, and $a_{i j}$ is the set of evaluation values for two decision-makers.

Table 1. Multi-hesitant fuzzy decision matrix.

\begin{tabular}{ccccc}
\hline & $c_{\mathbf{1}}$ & $\boldsymbol{c}_{\mathbf{2}}$ & $c_{\mathbf{3}}$ & $\boldsymbol{c}_{\mathbf{4}}$ \\
\hline$a_{1}$ & $\{0.4,0.5,0.7\}$ & $\{0.5,0.5,0.8\}$ & $\{0.6,0.6,0.9\}$ & $\{0.5,0.6\}$ \\
$a_{2}$ & $\{0.6,0.7,0.8\}$ & $\{0.5,0.6\}$ & $\{0.6,0.7,0.7\}$ & $\{0.4,0.5\}$ \\
$a_{3}$ & $\{0.6,0.8\}$ & $\{0.2,0.3,0.5\}$ & $\{0.6,0.6\}$ & $\{0.5,0.7\}$ \\
$a_{4}$ & $\{0.5,0.5,0.7\}$ & $\{0.4,0.5\}$ & $\{0.8,0.9\}$ & $\{0.3,0.4,0.5\}$ \\
$a_{5}$ & $\{0.6,0.7\}$ & $\{0.5,0.7\}$ & $\{0.7,0.8\}$ & $\{0.3,0.3,0.4\}$ \\
\hline
\end{tabular}

\subsection{An Illustration of the Proposed Approach}

There are four cases that the proposed approach is used to handle the MCDM problems where the weight of criteria is known or unknown. The procedures of obtaining the optimal alternative, by using the developed approach, are shown as follows.

Case 1. If the ordered positions of the multi-hesitant fuzzy values of criteria are considered, then the GMHFOWA operator is utilized and the associated weight is $w=(0.33,0.18,0.37,0.12)$. The procedures of the proposed approach can be obtained.

Step 1. Normalize the data in Table 1.

Because all the criteria are of the maximizing type and have the same measurement unit, there is no need for normalization and $\widetilde{R}=\left(\widetilde{a}_{i j}\right)_{5 \times 4}=\left(a_{i j}\right)_{5 \times 4}$.

Steps 2-3. Aggregate the MHFNs of each decision-maker and calculate the score function value and accuracy function value.

According to Definition 5 and Theorem 1, the following results can be obtained:

$$
s\left(y_{1}\right)=0.5810 ; s\left(y_{2}\right)=0.5782 ; s\left(y_{3}\right)=0.5240 ; s\left(y_{4}\right)=0.5247 ; s\left(y_{5}\right)=0.5485
$$

Since the score function values are different, so there is no need to compute the accuracy function value.

Step 4. Rank the alternatives.

Based on Step 3, since $s\left(y_{3}\right)<s\left(y_{4}\right)<s\left(y_{5}\right)<s\left(y_{2}\right)<s\left(y_{1}\right)$, so the final ranking is $a_{3} \prec a_{4} \prec$ $a_{5} \prec a_{2} \prec a_{1}$. The best alternative is $a_{1}$ while the worst alternative is $a_{3}$.

Case 2. If both the multi-hesitant fuzzy values of criteria and their ordered positions are considered, then the GMHFHWA operator is utilized. If the corresponding vector of criteria is $w=(0.33,0.18,0.37,0.12)$ and the aggregation-associated vector is $\omega=(0.3,0.25,0.2,0.25)$, according Definition 6 and Theorem 2, the following results can be obtained:

$$
s\left(y_{1}\right)=0.3207 ; s\left(y_{2}\right)=0.3583 ; s\left(y_{3}\right)=0.2940 ; s\left(y_{4}\right)=0.3098 ; s\left(y_{5}\right)=0.3750
$$

Since $s\left(y_{3}\right)<s\left(y_{4}\right)<s\left(y_{1}\right)<s\left(y_{2}\right)<s\left(y_{5}\right)$, so the final ranking is $a_{3} \prec a_{4} \prec a_{1} \prec a_{2} \prec a_{5}$. The best alternative is $a_{5}$ while the worst alternative is $a_{3}$. 
Case 3. If the prioritization among the multi-hesitant fuzzy values of criteria is taken into account, then the GMHFPWA operator is used and the prioritization relation for criteria is $c_{1} \succ c_{2} \succ c_{3} \succ c_{4}$. Based on Definition 7 and Theorem 3,

$$
\begin{gathered}
T_{11}=1, T_{12}=s\left(a_{11}\right)=0.5300, T_{13}=0.3180, T_{14}=0.2226, \sum_{j=1}^{4} T_{j}=2.0706 ; \\
T_{21}=1, T_{22}=s\left(a_{21}\right)=0.7000, T_{23}=0.3850, T_{24}=0.2568, \sum_{j=1}^{4} T_{j}=2.3418 ; \\
T_{31}=1, T_{32}=s\left(a_{31}\right)=0.7000, T_{33}=0.2310, T_{34}=0.1386, \sum_{j=1}^{4} T_{j}=2.0696 ; \\
T_{41}=1, T_{42}=s\left(a_{41}\right)=0.5670, T_{43}=s\left(a_{41}\right) \times s\left(a_{42}\right)=0.5670 \times 0.4500=0.2552, \\
T_{44}=s\left(a_{41}\right) \times s\left(a_{42}\right) \times s\left(a_{44}\right)=0.5670 \times 0.4500 \times 0.8500=0.2169, \sum_{j=1}^{4} T_{j}=2.0391 ; \\
T_{51}=1, T_{52}=s\left(a_{51}\right)=0.6500, T_{53}=0.3900, T_{54}=0.2925, \sum_{j=1}^{4} T_{j}=2.3325 .
\end{gathered}
$$

Therefore, the following results can be obtained:

$$
s\left(y_{1}\right)=0.5787 ; s\left(y_{2}\right)=0.6223 ; s\left(y_{3}\right)=0.5581 ; s\left(y_{4}\right)=0.5520 ; s\left(y_{5}\right)=0.6131
$$

Since $s\left(y_{4}\right)<s\left(y_{3}\right)<s\left(y_{1}\right)<s\left(y_{5}\right)<s\left(y_{2}\right)$, so the final ranking is $a_{4} \prec a_{3} \prec a_{1} \prec a_{5} \prec a_{2}$. The best alternative is $a_{2}$ while the worst alternative is $a_{4}$.

Case 4. If the correlations among the multi-hesitant fuzzy values of criteria are considered, then the GMHFCIWA operator can be used. Based on Definition 8 and Theorem 4, suppose $\mu\left(c_{1}\right)=0.40$, $\mu\left(c_{2}\right)=0.25, \mu\left(c_{3}\right)=0.37, \mu\left(c_{4}\right)=0.20, \mu\left(c_{1}, c_{2}\right)=0.60, \mu\left(c_{1}, c_{3}\right)=0.70, \mu\left(c_{1}, c_{4}\right)=0.56$, $\mu\left(c_{2}, c_{3}\right)=0.68, \mu\left(c_{2}, c_{4}\right)=0.43, \mu\left(c_{3}, c_{4}\right)=0.54, \mu\left(c_{1}, c_{2}, c_{3}\right)=0.88, \mu\left(c_{1}, c_{2}, c_{4}\right)=0.75$, $\mu\left(c_{2}, c_{3}, c_{4}\right)=0.73, \mu\left(c_{1}, c_{3}, c_{4}\right)=0.84$, and $\mu\left(c_{1}, c_{2}, c_{3}, c_{4}\right)=1$, then the following results can be obtained.

$$
s\left(y_{1}\right)=0.6200 ; s\left(y_{2}\right)=0.6320 ; s\left(y_{3}\right)=0.6050 ; s\left(y_{4}\right)=0.6305 ; s\left(y_{5}\right)=0.6400
$$

Since $s\left(y_{3}\right)<s\left(y_{1}\right)<s\left(y_{4}\right)<s\left(y_{2}\right)<s\left(y_{5}\right)$, so the final ranking is $a_{3} \prec a_{1} \prec a_{4} \prec a_{2} \prec a_{5}$. The best alternative is $a_{5}$ while the worst alternative is $a_{3}$.

\subsection{Sensitivity Analysis}

In Step 2, four aggregation operators can be used, and the sensitivity analysis will be conducted in these cases. Since the aggregation parameter $\lambda$ is a balance factor, which can be determined by decision-makers based on their preference. To investigate the influence of different $\lambda$ on the ranking of alternatives, various $\lambda$ are utilized. If the GMHFOWA operator, the GMHFHWA operator, the GMHPWA operator and the GMHCIWA operator are used respectively, then the ranking results are shown in Tables 2 and 3.

Table 2. Rankings obtained using the GMHFOWA operator and the GMHFHWA operator.

\begin{tabular}{ccc}
\hline \multirow{2}{*}{$\lambda$} & RMHFOWA \\
\cline { 2 - 3 } & GMHFS \\
\hline$\lambda=1$ & $a_{3} \prec a_{4} \prec a_{5} \prec a_{2} \prec a_{1}$ & $a_{3} \prec a_{4} \prec a_{1} \prec a_{2} \prec a_{5}$ \\
$\lambda=2$ & $a_{3} \prec a_{4} \prec a_{5} \prec a_{2} \prec a_{1}$ & $a_{3} \prec a_{4} \prec a_{1} \prec a_{2} \prec a_{5}$ \\
$\lambda=5$ & $a_{3} \prec a_{4} \prec a_{5} \prec a_{2} \prec a_{1}$ & $a_{3} \prec a_{4} \prec a_{1} \prec a_{2} \prec a_{5}$ \\
$\lambda=10$ & $a_{3} \prec a_{4} \prec a_{5} \prec a_{2} \prec a_{1}$ & $a_{3} \prec a_{4} \prec a_{1} \prec a_{2} \prec a_{5}$ \\
\hline
\end{tabular}


Table 3. Rankings obtained using the GMHFPWA and the GMHFCIWA operators.

\begin{tabular}{|c|c|c|}
\hline \multirow{2}{*}{$\lambda$} & \multicolumn{2}{|c|}{ Rankings } \\
\hline & GMHFPWA & GMHFCIWA \\
\hline$\lambda=1$ & $a_{4} \prec a_{3} \prec a_{1} \prec a_{5} \prec a_{2}$ & $a_{3} \prec a_{1} \prec a_{4} \prec a_{2} \prec a_{5}$ \\
\hline$\lambda=2$ & $a_{4} \prec a_{3} \prec a_{1} \prec a_{5} \prec a_{2}$ & $a_{3} \prec a_{1} \prec a_{4} \prec a_{2} \prec a_{5}$ \\
\hline$\lambda=5$ & $a_{4} \prec a_{3} \prec a_{1} \prec a_{5} \prec a_{2}$ & $a_{3} \prec a_{1} \prec a_{4} \prec a_{2} \prec a_{5}$ \\
\hline$\lambda=10$ & $a_{4} \prec a_{3} \prec a_{1} \prec a_{5} \prec a_{2}$ & $a_{3} \prec a_{1} \prec a_{4} \prec a_{2} \prec a_{5}$ \\
\hline
\end{tabular}

From Tables 2 and 3, it can be seen that if the GMHFCIWA operator and the GMHFHWA operator are used respectively in Step 2, then the final ranking is $a_{3} \prec a_{1} \prec a_{4} \prec a_{2} \prec a_{5}$ or $a_{3} \prec a_{4} \prec$ $a_{1} \prec a_{2} \prec a_{5}$. The best alternative is always $a_{5}$ while the worst alternative is $a_{3}$. If the GMHFOWA operator, the GMHFPWA operator are used respectively in Step 2, then the final ranking is $a_{3} \prec a_{4} \prec$ $a_{5} \prec a_{2} \prec a_{1}$ and $a_{4} \prec a_{3} \prec a_{1} \prec a_{5} \prec a_{2}$. The best alternative is $a_{1}$ or $a_{2}$ while the worst alternative is $a_{3}$ or $a_{4}$. However, for each operator, the rankings obtained are consistent as $\lambda$ changes. Moreover, for different operator, the aggregation parameter $\lambda$ also lead to different aggregation results, but the final rankings of alternatives are the same as the parameter changes. Moreover, different aggregation operators can be chosen according to the practical necessity of MCDM problems, which can represent the decision-makers' preference.

\subsection{A Comparison Analysis and Discussion}

In this section, to validate the feasibility of the proposed multi-hesitant fuzzy MCDM approach based on convex operators, a comparative study was conducted with other methods as shown in $\mathrm{Xu}$ [9,10], Zhang et al. [13], Yu [16], Zhang and Wei [31], Zhang and Xu [32], and Peng et al. [35]. Moreover, the method in Wei [11] considering the prioritization among criteria is also compared.

The method presented in Peng et al. [35] can deal with multi-hesitant fuzzy information directly. However, in other compared methods, they all do not clarify that how to solve a situation where there is a repeated value in the evaluation of alternatives. The comparison analysis was based on the same illustrative example, but the same value will be counted only once in Table 1 . Suppose the weight vector of criteria is $w=(0.33,0.18,0.37,0.12)$, then the compared results can be obtained as shown in Table 4 .

Table 4. Comparison of different methods.

\begin{tabular}{|c|c|c|}
\hline \multicolumn{2}{|c|}{ Methods } & Ranking of Alternatives \\
\hline \multicolumn{2}{|c|}{$\mathrm{Xu}[9,10]$} & $a_{3} \prec a_{2} \prec a_{1} \prec a_{5} \prec a_{4}$ \\
\hline \multicolumn{2}{|c|}{ Wei [11] } & $a_{3} \prec a_{1} \prec a_{4} \prec a_{5} \prec a_{2}$ \\
\hline \multicolumn{2}{|c|}{ Zhang [13] } & $a_{3} \prec a_{4} \prec a_{1} \prec a_{2} \prec a_{5}$ \\
\hline \multicolumn{2}{|c|}{$\mathrm{Yu}[16]$} & $a_{3} \prec a_{2} \prec a_{5} \prec a_{4} \prec a_{1}$ \\
\hline \multicolumn{2}{|c|}{ Zhang and Wei [31] } & $a_{3} \prec a_{4} \prec a_{2} \prec a_{1} \prec a_{5}$ \\
\hline \multicolumn{2}{|c|}{ Zhang and $\mathrm{Xu}$ [32] } & $a_{4} \prec a_{3} \prec a_{2} \prec a_{1} \prec a_{5}$ \\
\hline \multicolumn{2}{|c|}{ Peng et al. [35] } & $a_{4} \prec a_{3} \prec a_{2} \prec a_{1} \prec a_{5}$ \\
\hline \multirow{3}{*}{$\begin{array}{l}\text { Proposed } \\
\text { methods: }\end{array}$} & GMHFOWA & $a_{3} \prec a_{4} \prec a_{5} \prec a_{2} \prec a_{1}$ \\
\hline & GMHFHWA & $a_{3} \prec a_{4} \prec a_{1} \prec a_{2} \prec a_{5}$ \\
\hline & GMHFPWA & $a_{4} \prec a_{3} \prec a_{1} \prec a_{5} \prec a_{2}$ \\
\hline
\end{tabular}

According to the results presented in Table 4, the following conclusions can be categorically drawn. Firstly, the repetitive values in HFSs are not taken into consideration in the existing methods. Secondly, compared with the methods relying on aggregation operators, the result of using the GMHFHWA operator is the same as that using the method of Zhang [13], and the best alternative is always $a_{5}$ while the worst alternative is always $a_{3}$; the result of using the GMHFOWA operator is the same as that using the method of $\mathrm{Yu}$ [16] and the best alternative is $a_{1}$ while the worst alternative is $a_{3}$; However, the results of the proposed approach are different from that using the method of 
$\mathrm{Xu}[9,10]$. Furthermore, the method of Wei [11] and the proposed GMHFPWA operator are all considered the prioritization among criteria. However, there exist a litter difference between the result of using GMHFPWA operator and the result of using the method of Wei [11], the final ranking is $a_{4} \prec a_{3} \prec a_{1} \prec a_{5} \prec a_{2}$ or $a_{3} \prec a_{1} \prec a_{4} \prec a_{5} \prec a_{2}$. The best alternative is always $a_{2}$ while the worst alternative is $a_{4}$ or $a_{3}$. Apparently, different operations and aggregation operators being involved in those methods can interpret the differences existing in the final rankings to some extent. Thirdly, compared with the methods relying on distance measures, the result of using the GMHFWA operator or the GMHFHWA operator is the same as that using the method of Zhang and Wei [31] and the best alternative is always $a_{5}$ while the worst alternative is always $a_{3}$. However, it is different from that using of Zhang and $\mathrm{Xu}$ [32] that the best alternative is $a_{5}$ while the worst alternative is $a_{4}$. Furthermore, the methods using distance measures have certain shortcomings because the condition should be satisfied that all HFNs must be arranged in ascending order and be of equal length. If two HFNs being compared have different lengths, then the value of the shorter one should be increased subjectively until both are equal. Finally, the result using the method of Peng et al. [35] is the same as that of the proposed approach. Therefore, the proposed method can effectively overcome the shortcomings of the compared methods and the computation is very simple.

From the analysis above, it can be seen that the main advantages of the approach developed in this paper over the other methods are not only due to its ability to effectively deal with the preference information expressed by MHFNs, but also due to its consideration that the weight of criteria is known or unknown. This can avoid losing and distorting the preference information provided, which makes the results better correspond with real life decision-making problems.

\section{Conclusions}

HFSs are considered useful in handling decision-making problems under uncertain situations where decision-makers hesitate when choosing between several values before expressing their preferences about weights and data. MHFSs can deal effectively with the case where some values are repeated more than once in an HFS. In this paper, the convex combination of MHFNs was discussed and some aggregation operators based on convex operation, such as GMHFOWA operator, GMHFHWA operator, GMHFPWA operator and GMHFCIWA operator, were developed as well. Moreover, a novel approach based on convex operators was developed to deal with MCDM problems where the data are MHFNs. Finally, an illustrative example was given to verify the proposed approach. The primary characteristic of the proposed approach is that those aggregation operators can provide more choices for decision-makers according to the actual decision-making environment. Moreover, MHFSs could overcome the shortcomings in HFSs where if two or more decision-makers set the same value, it is only counted once. Further research will investigate how to obtain the optimal values of criteria by a specified model within a multi-hesitant fuzzy environment.

Author Contributions: Conceptualization and Methodology, Y.M. and J.P.; Formal Analysis, J.P.; Validation, J.Y.; Original Draft Preparation, Y.M. and J.P.; Writing-Review \& Editing, J.Y.

Funding: This work was supported by the National Natural Science Foundation, P.R. China (No. 71701065) and Educational Commission of Guangdong Province of China (2018JKZ022).

Acknowledgments: The authors thank the editors and anonymous reviewers for their helpful comments and suggestions.

Conflicts of Interest: The authors declare no conflict of interest.

\section{References}

1. Torra, V. Hesitant fuzzy sets. Int. J. Intell. Syst. 2010, 25, 529-539. [CrossRef]

2. Torra, V.; Narukawa, Y. On hesitant fuzzy sets and decision. In Proceedings of the 18th IEEE International Conference on Fuzzy Systems, Jeju Island, Korea, 20-24 August 2009; pp. 1378-1382.

3. Zadeh, L.A. Fuzzy sets. Inf. Control 1965, 8, 338-356. [CrossRef] 
4. Song, C.; Xu, Z.; Zhao, H. A novel comparison of probabilistic hesitant fuzzy elements in multi-criteria decision making. Symmetry 2018, 10, 177. [CrossRef]

5. Faizi, S.; Sałabun, W.; Rashid, T.; Watróbski, J.; Zafar, S. Group decision-making for hesitant fuzzy sets based on characteristic objects method. Symmetry 2017, 9, 136. [CrossRef]

6. Faizi, S.; Rashid, T.; Saabun, W.; Zafar, S.; Wtróbski, J. Decision making with uncertainty using hesitant fuzzy sets. Int. J. Fuzzy Syst. 2018, 20, 93-103. [CrossRef]

7. Liao, H.; Wu, D.; Huang, Y.; Ren, P.; Xu, Z.; Verma, M. Green logistic provider selection with a hesitant fuzzy linguistic thermodynamic method integrating cumulative prospect theory and PROMETHEE. Sustainability 2018, 10, 1291. [CrossRef]

8. Liu, P.; Gao, H. Multi-criteria decision making based on generalized Maclaurin symmetric means with multi-hesitant fuzzy linguistic information. Symmetry 2018, 10, 81. [CrossRef]

9. Xia, M.M.; Xu, Z.S. Hesitant fuzzy information aggregation in decision making. Int. J. Approx. Reason. 2011, 52, 395-407. [CrossRef]

10. Zhu, B.; Xu, Z.S.; Xia, M.M. Hesitant fuzzy geometric Bonferoni means. Inf. Sci. 2012, 205, 72-85. [CrossRef]

11. Wei, G.W. Hesitant fuzzy prioritized operators and their application to multiple attribute decision making. Knowl. Based Syst. 2012, 31, 176-182. [CrossRef]

12. Xia, M.M.; Xu, Z.S.; Chen, N. Some Hesitant fuzzy aggregation operators with their application in group decision making. Group Decis. Negot. 2013, 22, 259-279. [CrossRef]

13. Zhang, Z.M.; Wang, C.; Tian, D.Z.; Li, K. Induced generalized hesitant fuzzy operators and their application to multiple attribute group decision making. Comput. Ind. Eng. 2014, 67, 116-138. [CrossRef]

14. Zhou, W. An Accurate method for determining hesitant fuzzy aggregation operator weights and its application to project investment. Int. J. Intell. Syst. 2014, 29, 668-686. [CrossRef]

15. Zhang, Z.M. Hesitant fuzzy power aggregation operators and their application to multiple attribute group decision making. Inf. Sci. 2013, 234, 150-181. [CrossRef]

16. Yu, D.J. Some hesitant fuzzy information aggregation operators based on Einstein operational laws. Int. J. Intell. Syst. 2014, 29, 320-340. [CrossRef]

17. Chen, N.; Xu, Z.S.; Xia, M.M. Correlation coefficients of hesitant fuzzy sets and their applications to clustering analysis. Appl. Math. Model. 2013, 37, 2197-2211. [CrossRef]

18. Xu, Z.S.; Xia, M.M. Distance and similarity measures for hesitant fuzzy sets. Inf. Sci. 2011, 181, $2128-2138$. [CrossRef]

19. Xu, Z.S.; Xia, M.M. On distance and correlation measures of hesitant fuzzy information. Int. J. Intell. Syst. 2011, 26, 410-425. [CrossRef]

20. Farhadinia, B. Distance and similarity measures for higher order hesitant fuzzy sets. Knowl. Based Syst. 2014, 55, 43-48. [CrossRef]

21. Wang, L.; Ni, M.F.; Yu, Z.K.; Zhu, L. Power geometric operators of hesitant multiplicative fuzzy numbers and their application to multiple attribute group decision making. Math. Probl. Eng. 2014, 2014, 186502. [CrossRef]

22. Torres, R.; Salas, R.; Astudillo, H. Time-based hesitant fuzzy information aggregation approach for decision-making problems. Int. J. Intell. Syst. 2014, 29, 579-595. [CrossRef]

23. Qian, G.; Wang, H.; Feng, X.Q. Generalized hesitant fuzzy sets and their application in decision support system. Knowl. Based Syst. 2013, 37, 357-365. [CrossRef]

24. Meng, F.Y.; Chen, X.H.; Zhang, Q. Induced generalized hesitant fuzzy Shapley hybrid operators and their application in multi-attribute decision making. Appl. Soft Comput. 2015, 28, 599-607. [CrossRef]

25. Zhou, W.; Xu, Z.S. Optimal discrete fitting aggregation approach with hesitant fuzzy information. Knowl. Based Syst. 2015, 78, 22-33. [CrossRef]

26. Tan, C.Q.; Yi, W.T.; Chen, X.H. Hesitant fuzzy Hamacher aggregation operators for multicriteria decision making. Appl. Soft Comput. 2015, 26, 325-349. [CrossRef]

27. Meng, F.Y.; Chen, X.H. Correlation coefficients of hesitant fuzzy sets and their application based on fuzzy measures. Cognative Comput. 2015, 7, 445-463. [CrossRef]

28. Liao, H.C.; Xu, Z.S.; Zeng, X.J. Novel correlation coefficients between hesitant fuzzy sets and their application in decision making. Knowl. Based Syst. 2015, 82, 115-127. [CrossRef]

29. Li, D.Q.; Zeng, W.Y.; Li, J.H. New distance and similarity measures on hesitant fuzzy sets and their applications in multiple criteria decision making. Eng. Appl. Artif. Intell. 2015, 40, 11-16. [CrossRef] 
30. Hu, J.H.; Zhang, X.L.; Chen, X.H.; Liu, Y.M. Hesitant fuzzy information measures and their applications in multi-criteria decision making. Int. J. Syst. Sci. 2015, 87, 91-103. [CrossRef]

31. Zhang, N.; Wei, G.W. Extension of VIKOR method for decision making problem based on hesitant fuzzy set. Appl. Math. Model. 2013, 37, 4938-4947. [CrossRef]

32. Zhang, X.L.; Xu, Z.S. The TODIM analysis approach based on novel measured functions under hesitant fuzzy environment. Knowl. Based Syst. 2014, 61, 48-58. [CrossRef]

33. Farhadinia, B. A novel method of ranking hesitant fuzzy values for multiple attribute decision-making problems. Int. J. Intell. Syst. 2013, 28, 752-767. [CrossRef]

34. Farhadinia, B. Information measures for hesitant fuzzy sets and interval-valued hesitant fuzzy sets. Inf. Sci. 2013, 240, 129-144. [CrossRef]

35. Peng, J.J.; Wang, J.Q.; Wang, J.; Yang, L.J.; Chen, X.H. An extension of ELECTRE to multi-criteria decision-making problems with multi-hesitant fuzzy sets. Inf. Sci. 2015, 307, 113-126. [CrossRef]

36. Chen, N.; Xu, Z.S. Hesitant fuzzy ELECTRE II approach: A new way to handle multi-criteria decision making problems. Inf. Sci. 2015, 292, 175-197. [CrossRef]

37. Yager, R.R. Prioritized aggregation operators. Int. J. Approx. Reason. 2008, 48, 263-274. [CrossRef]

38. Wang, Z.; Klir, G.J. Fuzzy Measure Theory; Plenum Press: New York, NY, USA, 1992.

39. Schmeidler, D. Subjective probability and expected utility without additivity. Econometrica 1989, 57, 517-587. [CrossRef]

(C) 2018 by the authors. Licensee MDPI, Basel, Switzerland. This article is an open access article distributed under the terms and conditions of the Creative Commons Attribution (CC BY) license (http://creativecommons.org/licenses/by/4.0/). 